\title{
Unilateral Pure Trigeminal Motor Neuropathy
}

\author{
Rosy Bala ${ }^{1}$, Nitin Gupta 2 , Sandeep Joshi ${ }^{3}$ \\ ${ }^{1}$ Department of Microbiology, MMIMSR, Mullana (MMDU), Ambala, Haryana, India. \\ 2Department of Medicine, MMIMSR, Mullana (MMDU), Ambala, Haryana, India. \\ ${ }^{3}$ Department of General Medicine, MMIMSR, Mullana (MMDU), Ambala, Haryana, India.
}

\section{PRESENTATION OF CASE}

A 17-year-old male reported to us in the Department of Medicine, Maharishi Markandeshwar Institute of Medical Sciences \& Research, Mullana, Ambala, Haryana, India, in July 2019 with a complaint of left sided facial asymmetry since one and half years which was insidious in onset. The patient was himself unaware of the facial asymmetry and it was initially noticed by his mother. Gradually, the asymmetry of face became more prominent and was noticed by the patient and other relatives as well. There was no history of pain, numbness, difficulty in chewing or mouth opening, swallowing of food. He had no preceding febrile illness or local trauma. On local examination, there was no tenderness on left side of face. There was visible asymmetry of face due to atrophy of left masseter muscle [figure 1]. No sensory deficit was elicited during neurological examination. The diagnosis of unilateral pure trigeminal motor neuropathy was made clinically. Confirmation of the neuropathy was dine using Electromyography study which showed some fibrillation potentials and positive sharp waves in the muscles innervated by trigeminal nerve (masseter), however nerve conduction study was normal. In our patient, the MRI Brain (done at other centre) didn't show any intracranial abnormality and was inconclusive to diagnose atrophy and fat deposition in masseter muscle. Demonstration of fat deposition and atrophy of masseter muscle on MRI Face would have been diagnostic in this case but it could not be obtained as consent for repeat MRI was not given by the patient. No nerve/muscle biopsy was undertaken in the present case. The diagnosis was primarily on the basis of clinical examination and collaborative EMG findings. To rule out these aetiologies, radiological evaluation of the patient including MRI Brain is indicated.

There is no definite etiological factor associated with pure motor trigeminal neuropathy, although there are reports of its association with following underlying disorders:

1. Infectious (Viral).

2. Autoimmune (RA, Sjogren Syndrome).

3. Vascular (Infarction).

4. Neoplastic (Meningioma).

5. Multiple Sclerosis.

6. Trauma.
Corresponding Author: Dr. Nitin Gupta, Assistant Professor, Department of Medicine, MMIMSR Mullana (MMDU), Ambala, Haryana, India. E-mail:drnitintayal@gmail.com

DOI: $10.14260 /$ jemds/2020/37

Financial or Other Competing Interests: None.

How to Cite This Article:

Bala R, Gupta N, Joshi S. Unilateral pure trigeminal motor neuropathy. J. Evolution Med. Dent. Sci. 2020;9(03):166-167, DOI: 10.14260/jemds/2020/37

Submission 31-11-2019,

Peer Review 01-01-2020,

Acceptance 08-01-2020,

Published 20-01-2020. 


\section{DISCUSSION OF MANAGEMENT}

Since the patient didn't have any complaints apart from the asymmetry of face, he was counselled regarding his neurological condition and need of regular follow-up.

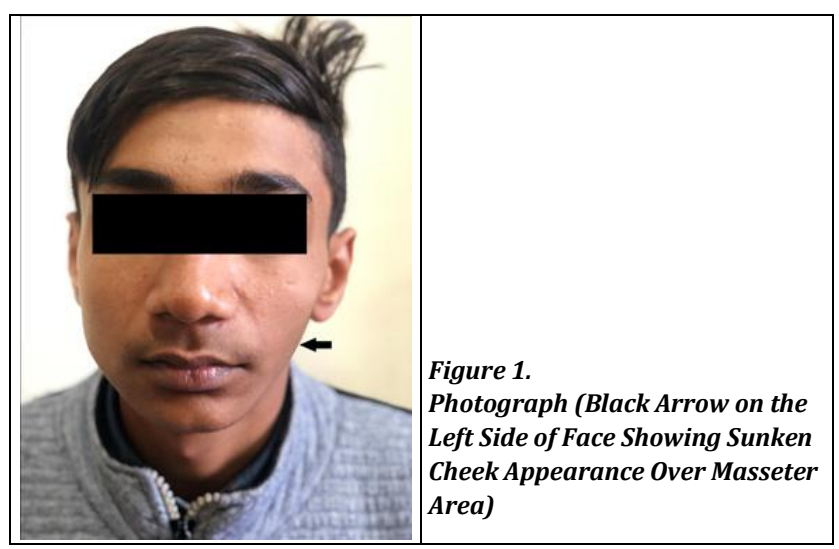

\section{DISCUSSION}

Pure trigeminal nerve motor neuropathy is a clinical rarity with no estimated incidence or prevalence in literature. Although the condition was first described by Chia LG et al in $1988,[1]$ but to the best of our knowledge, very few cases have been reported in literature till date. UTMN is characterized by mandibular branch motor weakness without any signs of trigeminal sensory or other cranial nerve involvement. The underlying causes reported include infections, cerebrovascular accident, neoplastic or traumatic events. [2] The clinical manifestation varies with the chronicity of the process, like facial asymmetry, pain, weakness on chewing. Opening deviation is one of the important symptoms of pure trigeminal motor neuropathy. ${ }^{[3]}$ In some patients, there may be initial symptoms like mastication problems and feeling of weakness in the jaw muscles, most of patients were reported after visible asymmetry of the face was recognized by the patient or some relative. In the present case also, the facial asymmetry was first recognized by the mother of the patient. The trigeminal nerve has four brainstem nuclei: the main sensory nucleus, the spinal nucleus, the motor nucleus, and the mesencephalic nucleus, which is involved in proprioception. The motor nucleus of the trigeminal nerve is located medial to the principal sensory nucleus in the rostral pons. The large sensory and smaller motor roots exit via the lateral pons as a common trunk and the motor root remains inferior to the sensory root through the course in the preganglionic segment and the trigeminal ganglion. The trigeminal ganglion lies in Meckel's cave, and distal to the ganglion, the trigeminal nerve trifurcates into its three principal branches, the ophthalmic (V1), maxillary (V2), and mandibular nerves (V3).[4] Any lesion which involves the fifth cranial nerve along its protracted course from its nucleus in the brainstem to its distal facial ramifications to the masticatory muscles may result in the features of trigeminal motor involvement. Selective involvement of the motor fibres may be possibly caused by inflammatory, infectious, and post viral autoimmune processes.[5]

Diagnosis of pure motor trigeminal neuropathy involves clinical neurological examination, radiological evaluation including MRI (which may be help in differential diagnosis) and Electromyographic evaluation. There is no effective treatment for this condition. The management may include surgical intervention in cases in whom definite underlying pathological cause like a tumour is located on radiological evaluation. In other patients where no aetiology is confirmed, the treatment of pure motor trigeminal neuropathy includes reassurance and physiotherapy, although some attempts have been made to surgically correct the disability. ${ }^{[3]}$

\section{FINAL DIAGNOSIS}

The final diagnosis of pure motor trigeminal neuropathy was made clinically. There was no sensory involvement. Investigations didn't reveal any underlying secondary aetiology for the disorder.

\section{REFERENCES}

[1] Chia LG. Pure trigeminal motor neuropathy. Br Med J (Clin Res Ed) 1988;296(6622):609-10.

[2] Kämppi A, Kämppi L, Kemppainen P, et al. Focal atrophy of the unilateral masticatory muscles caused by pure trigeminal motor neuropathy: case report. Clin Case Rep 2018;6(5):939-43.

[3] Chai Y, Wei W, Chen M, et al. Surgical therapy for pure trigeminal motor neuropathy accompanied by limited mouth opening: a retrospective study. Dentistry 2015;5(10):337.

[4] Kerr FWL. The divisional organization of afferent fibres of the trigeminal nerve. Brain 1963;86(4):721-32.

[5] Park KS, Chung JM, Jeon BS, et al. Unilateral trigeminal mandibular motor neuropathy caused by tumor in the foramen Ovale. J Clin Neurol 2006;2(3):194-7. 